Mathematical Research Letters 1, 677-688 (1994)

\title{
COMPARISONS OF LAPLACE SPECTRA, LENGTH SPECTRA AND GEODESIC FLOWS OF SOME RIEMANNIAN MANIFOLDS
}

\author{
Carolyn Gordon and Yiping MaO
}

\section{Introduction}

The goal of this article is to discuss relationships between the Laplace spectrum, the length spectrum and the geodesic flow of compact Riemannian manifolds. We will say two compact Riemannian manifolds $M_{1}$ and $M_{2}$ are Laplace isospectral if the associated Laplace-Beltrami operators, acting on smooth functions, have the same eigenvalue spectrum. We will say they have $C^{k}$-conjugate geodesic flows if there exists a $C^{k}$-diffeomorphism $F: S\left(M_{1}\right) \rightarrow S\left(M_{2}\right)$ between the unit tangent bundles which interwines the geodesic flows of $M_{1}$ and $M_{2}$.

Since the Laplacian may be viewed as the quantum analogue of the classical dynamics, i.e., the geodesic flow, one might expect that Laplace isospectral manifolds would have conjugate geodesic flows. However, this is not the case. For example, flat tori with $C^{0}$-conjugate geodesic flows must be isometric, whereas examples of Laplace isospectral, non-isometric flat tori are well-known ([7], [18]). Similarly, C. Croke ([8]) and J. Otal ([19]) have independently shown that negatively curved surfaces with $C^{1}$ conjugate geodesic flows must be isometric, whereas examples of Laplace isospectral, non-isometric Riemann surfaces have been constructed in every genus $\geq 4$ (see [4], [5]). As we will discuss below, even continuous families of Laplace isospectral manifolds need not have $C^{0}$-conjugate geodesic flows.

The length spectrum of a compact Riemannian manifold $M$ is the collection of lengths of closed geodesics, counted with multiplicities. We define the multiplicity of a length to be the number of free homotopy classes of closed curves containing a geodesic of the given length. (Other notions

1991 Mathematics Subject Classification. 58G25, 53C22.

Key words and phrases. Laplace spectrum, length spectrum, geodesic flow.

Received August 15, 1994.

The first author was supported in part by NSF grant \#DMS 9296266. Research at MSRI is supported by NSF grant \#DMS 9022140. 
of multiplicity also appear in the literature.) We will call the collection of lengths, without multiplicities, the weak length spectrum. For generic manifolds, the Laplace spectrum determines the length spectrum ([6], [10]). However, the known examples of Laplace isospectral manifolds are far from generic, and it is interesting to compare their length spectra. All known examples have the same weak length spectrum; however, in some cases the multiplicities of the lengths differ (see [12], [14]).

Most known examples of Laplace isospectral manifolds are constructed by representation theoretic methods. All manifolds constructed by these methods are actually strongly isospectral, i.e., all natural strongly elliptic self-adjoint operators on the manifolds (e.g., the Hodge Laplacian on pforms) are isospectral.

In Section 1, we review the representation theoretic methods and explain why the resulting Laplace isospectral manifolds have the same weak length spectra. We also illustrate these ideas by proving a new result:

Theorem A. Compact orientable strongly isospectral hyperbolic manifolds always have the same length spectra.

We remark that every free homotopy class in a hyperbolic manifold contains a unique geodesic, so our notion of multiplicity agrees with all other notions in the literature. For Riemann surfaces, the Selberg trace formula shows that the Laplace spectrum determines the length spectrum; however in higher dimensions, one cannot read off the multiplicities from the Selberg trace formula. In case the length spectrum is simple (i.e., all lengths have multiplicities one), Colin de Verdière showed that the Laplace spectrum determines the length spectrum ([6]); see also [10]. The prime ingredient in Theorem A is a recent result of H. Pesce ([22]) showing that all strongly isospectral hyperbolic manifolds can be constructed by representation methods.

The primary emphasis of this article, however, is on nilmanifolds. These are among the simplest of the Riemannian manifolds, yet they have a very rich geometry. In Section 2, after reviewing the construction of Laplace isospectral deformations of nilmanifolds (again via representation theoretic methods) and discussing their length spectra, we announce the following results:

Theorem B. There exists a class $\mathcal{M}$ of two-step nilmanifolds such that any nilmanifold whose geodesic flow is $C^{0}$-conjugate to an element $M$ of $\mathcal{M}$ must be isometric to $M$. Included in $\mathcal{M}$ are continuous families of strongly Laplace isospectral manifolds.

A more precise statement is given in Section 2. Among the manifolds in $\mathcal{M}$ are all nilmanifolds of Heisenberg type. The class $\mathcal{M}$ is closed under 
finite coverings, so if two nilmanifolds $M_{1}$ and $M_{2}$, one of which is in $\mathcal{M}$, have finite covers with $C^{0}$-conjugate geodesic flows, then $M_{1}$ and $M_{2}$ must be isometric. The proof of Theorem B is fairly technical and is not included here. Instead we give a sketch in the simplest case in order to illustrate the geometric ideas.

\section{Representation theoretic techniques}

Given a Lie group $G$ and a uniform discrete subgroup $\Gamma$ (here uniform means that $\Gamma \backslash G$ is compact), define the quasi-regular representation $R_{\Gamma}$ of $G$ on $\mathbf{L}^{2}(\Gamma \backslash G)$ by $\left(R_{\Gamma}(a) f\right)(x)=f(x a)$. We will say two uniform discrete subgroups $\Gamma_{1}$ and $\Gamma_{2}$ of $G$ are representation equivalent if $R_{\Gamma_{1}}$ is unitarily equivalent to $R_{\Gamma_{2}}$.

The condition that $\Gamma_{1}$ and $\Gamma_{2}$ are representation equivalent can be restated in terms of a conjugacy relationship between $\Gamma_{1}$ and $\Gamma_{2}$ as follows: For $h \in G$, let $[h]_{G}$ denote its conjugacy class and let $C(h, G)$ denote its centralizer. If $G$ contains a uniform discrete subgroup $\Gamma$, then $C(\gamma, \Gamma) \backslash C(\gamma, G)$ is compact and hence $C(\gamma, G)$ is unimodular for all $\gamma \in \Gamma$. Note that $C(h, G)$ is conjugate to $C(\gamma, G)$ if $h \in[\gamma]_{G}$. Thus we can consistently choose a Haar measure on $C(h, G)$ for all $h \in[\gamma]_{G}$ so that the conjugation mapping $C\left(h_{1}, G\right)$ to $C\left(h_{2}, G\right)$ is measure-preserving when $h_{1}, h_{2} \in[\gamma]_{G}$.

1.1 Lemma. (see [1]) Two uniform discrete subgroups $\Gamma_{1}$ and $\Gamma_{2}$ of $G$ are representation equivalent if and only if for each $G$-conjugacy class $[h]_{G}$, we have $\alpha_{h}\left(\Gamma_{1}\right)=\alpha_{h}\left(\Gamma_{2}\right)$, where

$$
\alpha_{h}(\Gamma)=\sum_{[\gamma]_{\Gamma} \subset[h]_{G}} \operatorname{Vol}(C(\gamma, \Gamma) \backslash C(\gamma, G))
$$

Here the volume is computed using the Haar measures chosen above. In particular, $\Gamma_{1}$ and $\Gamma_{2}$ must intersect exactly the same $G$-conjugacy classes.

In case $G$ is finite, $\alpha_{h}(\Gamma)=\#\left([h]_{G} \bigcap \Gamma\right)$, so $\Gamma_{1}$ and $\Gamma_{2}$ are representation equivalent if and only if they meet each conjugacy class of $G$ in the same number of elements.

Sunada ([25]) gave an elegant method for constructing strongly Laplace isospectral manifolds with a common finite covering. A number of generalizations have followed (e.g., [1], [3], [23]). Most, but not all, known Laplace isospectral manifolds can be obtained by these methods. We state one generalization here.

1.2 Proposition. ([9]) Let $\Gamma_{1}$ and $\Gamma_{2}$ be uniform discrete representation equivalent subgroups of a Lie group $G$. Suppse $G$ acts by isometries on a Riemannian manifold $M$ in such a way that $M_{1}:=\Gamma_{1} \backslash M$ and $M_{2}:=$ 
$\Gamma_{2} \backslash M$ are well-defined compact manifolds (i.e., $\Gamma_{i}$ acts freely and properly discontinuously). Then $M_{1}$ and $M_{2}$, with the Riemannian metrics induced from $M$, are strongly Laplace isospectral.

Sunada's Theorem is the special case that $G$ is finite. Another special case - that $G$ is a connected Lie group and $M=G$ with a left-invariant Riemannian metric - is both elementary and classical.

We now compare the length spectra of the manifolds in Proposition 1.2. For simplicity here, we assume $M$ is simply-connected so that $\pi_{1}\left(M_{i}\right)=\Gamma_{i}$. Recall that free homotopy classes of closed curves in a manifold correspond to conjugacy classes of the fundamental group.

1.3 Proposition. With the hypotheses of Proposition 1.2, assume $M$ is simply-connected. Then $M_{1}$ and $M_{2}$ have the same weak length spectrum. If each G-conjugacy class meets $\Gamma_{1}$ and $\Gamma_{2}$ in the same number of $\Gamma_{1}$, (respectively $\Gamma_{2}$ ) conjugacy classes, then $M_{1}$ and $M_{2}$ have the same length spectrum.

Proof. Let $i, j \in\{1,2\}$. (We allow $i=j$.) Let $[\gamma]_{\Gamma_{i}}$ and $\left[\gamma^{\prime}\right]_{\Gamma_{j}}$ be conjugacy classes in $\Gamma_{i}$ and $\Gamma_{j}$ lying in the same $G$-conjugacy class, say $\gamma^{\prime}=a \gamma a^{-1}$. The free homotopy class $[\gamma]_{\Gamma_{i}}$ contains a geodesic of length $l$ if and only if there exists a geodesic $\sigma$ in $M$ such that $\sigma(t+l) \equiv \gamma \sigma(t)$. When this holds, $a \sigma$ is also a geodesic in $M$, since $a$ is an isometry. Moreover, $a \sigma(t+l) \equiv$ $\gamma^{\prime} \sigma(t)$. Thus $\left[\gamma^{\prime}\right]_{\Gamma_{j}}$ also contains a geodesic of length $l$. The proposition is now immediate from Lemma 1.1.

Perhaps surprisingly, $M_{1}$ and $M_{2}$ may not have the same length spectrum (with multiplicities) even when $M=G$ with a left-invariant metric. Indeed R. Gornet ([15]) has constructed counterexamples where $G$ is a simply-connected nilpotent Lie group.

We now turn to hyperbolic manifolds. Recently H. Pesce proved a converse to Proposition 1.2 in this case.

1.4 Proposition. ([22]) Let $M_{1}=\Gamma_{1} \backslash \mathbf{H}^{n}$ and $M_{2}=\Gamma_{2} \backslash \mathbf{H}^{n}$ be strongly isospectral compact orientable hyperbolic manifolds. Then $\Gamma_{1}$ and $\Gamma_{2}$ are representation equivalent subgroups of the full isometry group $G$ of $\mathbf{H}^{n}$.

We now use Lemma 1.1 and Propositions 1.3 and 1.4 to prove Theorem $\mathrm{A}$ in the introduction.

Recall that if $M=\Gamma \backslash \mathbf{H}^{n}$ is a compact hyperbolic manifold, then the centralizer $C(\gamma, \Gamma)$ is cyclic for all non-trivial $\gamma \in \Gamma$ (see [2]). We say $\gamma$ is prime if $C(\gamma, \Gamma)=\langle\gamma\rangle$. In general, $\gamma=\bar{\gamma}^{k}$ for some prime element $\bar{\gamma} \in \Gamma$ and $k \in \mathbf{Z}^{+}$and then $C(\gamma, \Gamma)=<\bar{\gamma}>$. Note that conjugates of prime elements are prime. Each free homotopy class $[\gamma]_{\Gamma}$ contains a unique 
closed geodesic; the geodesic is prime if and only if $\gamma$ is prime. We will for convenience refer to the length of $[\gamma]_{\Gamma}$ and write $l\left([\gamma]_{\Gamma}\right)$ to mean the length of this closed geodesic.

Now let $M_{1}=\Gamma_{1} \backslash \mathbf{H}^{n}$ and $M_{2}=\Gamma_{2} \backslash \mathbf{H}^{n}$ be strongly Laplace isospectral oriented compact hyperbolic manifolds. By Proposition 1.4, $\Gamma_{1}$ and $\Gamma_{2}$ are representation equivalent in $G$, and by the proof of Proposition 1.3, all $\Gamma_{1}$ and $\Gamma_{2}$-conjugacy classes lying in a given $G$-conjugacy class $[h]_{G}$ have the same length (which we will also refer to as the length of $[h]_{G}$ ). We must show $[h]_{G}$ intersects $\Gamma_{1}$ and $\Gamma_{2}$ in the same number of conjugacy classes.

Order the lengths in the weak length spectra of $M_{1}$ and $M_{2}$ (recall these are the same by 1.3) as $l_{1}<l_{2}<l_{3}<\cdots$. First suppose $[h]_{G}$ has length $l_{1}$. Then all $\Gamma_{i}$-conjugacy classes in $[h]_{G}$ must be prime. Let $[\gamma]_{\Gamma_{i}}$ and $\left[\gamma^{\prime}\right]_{\Gamma_{j}}$ be two such classes, say $\gamma^{\prime}=a \gamma a^{-1}$ with $a \in G$. (As before we allow $i, j \in\{1,2\}$ to be equal.) Since

$$
C\left(\gamma^{\prime}, \Gamma_{j}\right)=<\gamma^{\prime}>=<a \gamma a^{-1}>=a C\left(\gamma, \Gamma_{i}\right) a^{-1}
$$

and

$$
C\left(\gamma^{\prime}, G\right)=a C(\gamma, G) a^{-1}
$$

we have

$$
\operatorname{Vol}\left(C\left(\gamma, \Gamma_{i}\right) \backslash C(\gamma, G)\right)=\operatorname{Vol}\left(C\left(\gamma^{\prime}, \Gamma_{j}\right) \backslash C\left(\gamma^{\prime}, G\right)\right)
$$

Hence by Lemma 1.1, $[h]_{G}$ intersects $\Gamma_{1}$ and $\Gamma_{2}$ in the same number of conjugacy classes.

Now assume inductively that if $[h]_{G}$ has length less than $l_{j}$, then $[h]_{G}$ contains the same number of $\Gamma_{1}$ and $\Gamma_{2}$-conjugacy classes and the same number of prime $\Gamma_{1}$ and $\Gamma_{2}$-conjugacy classes (the second condition is actually a consequence of the first). Suppose that $[h]_{G}$ has length $l_{j}$. From the induction hypothesis, it follows that $[h]_{G}$ contains the same number of $k^{\text {th }}$ powers of prime $\Gamma_{1}$ and $\Gamma_{2}$ conjugacy classes for all $k \geqslant 2$. Moreover if $\gamma \in \Gamma_{1}$ and $\gamma^{\prime} \in \Gamma_{2}$ are $k^{\text {th }}$ powers of elements $\bar{\gamma} \in \Gamma_{1}$ and $\bar{\gamma}^{\prime} \in \Gamma_{2}$, conjugate in $G$, then

$$
\operatorname{Vol}\left(C\left(\gamma, \Gamma_{1}\right) \backslash C(\gamma, G)\right)=\operatorname{Vol}\left(C\left(\gamma^{\prime}, \Gamma_{2}\right) \backslash C\left(\gamma^{\prime}, G\right)\right) .
$$

Thus subtracting these contributions from $\alpha_{h}\left(\Gamma_{1}\right)$ and $\alpha_{h}\left(\Gamma_{2}\right)$ (in the notation of Lemma 1.1), we see that the sum of the remaining terms, corresponding to the prime conjugacy classes, must match. Next arguing as in the initial step, we see that $[h]_{G}$ contains the same number of prime $\Gamma_{1}$ and $\Gamma_{2}$ conjugacy classes. Theorem A now follows from Proposition 1.3. 


\section{Geodesic flow on nilmanifolds}

Let $G$ be a simply-connected nilpotent Lie group, $g$ a left-invariant Riemannian metric on $G$, and $\Gamma$ a uniform discrete subgroup of $G$. The compact Riemannian manifold ( $\Gamma \backslash G, g$ ) is called a nilmanifold. If $G$ is k-step nilpotent, we say $\Gamma \backslash G$ is a k-step nilmanifold. See [11] for a discussion of the geometry of nilmanifolds.

We first review conditions for two nilmanifolds to be Laplace isospectral.

2.1 Definition. Let $\Gamma$ be a uniform discrete subgroup of a simply-connected nilpotent Lie group $G$. An automorphism $\Phi$ of $G$ is said to be $\Gamma$-almost inner if $\Phi(\gamma)$ is conjugate to $\gamma$ for all $\gamma \in \Gamma$. The automorphism is said to be almost inner if $\Phi(x)$ is conjugate to $x$ for all $x \in G$.

The $\Gamma$-almost inner automorphisms and the almost inner automorphisms form connected Lie subgroups of $G$. In many cases these groups properly contain the group $\operatorname{Inn}(G)$ of inner automorphisms.

2.2 Proposition. ([12], [13]) Let $(\Gamma \backslash G, g)$ be a nilmanifold and $\Phi$ a $\Gamma$ almost inner automorphism. Then $\Gamma$ and $\Phi(\Gamma)$ are representation equivalent subgroups of $G$ and thus $(\Phi(\Gamma) \backslash G, g)$ and $(\Gamma \backslash G, g)$ are strongly Laplace isospectral. Moreover, they have the same length spectrum. If $\left\{\Phi_{t}\right\}_{t}$ is a continuous family of $\Gamma$-almost inner automorphisms not lying in $\operatorname{Inn}(G)$, then $\left(\Phi_{t}(\Gamma) \backslash G, g\right)_{t}$ is a non-trivial deformation of $(\Gamma \backslash G, g)$.

Note that $(\Phi(\Gamma) \backslash G, g)$ is isometric to $(\Gamma \backslash G, g)$, so the deformations may be viewed as deformations of metrics on the same underlying manifold.

In case $G$ is two-step nilpotent, H. Ouyang and H. Pesce ([20], [21]) independently proved conversely that every Laplace isospectral deformation $\left(\Gamma \backslash G, g_{t}\right)$, where the $g_{t}$ lift to left-invariant metrics on $G$, arises from a family of $\Gamma$-almost inner automorphisms.

2.3 Definition. Two Riemannian manifolds $M_{1}$ and $M_{2}$ are said to have the same marked length spectrum if there exists an isomorphism $\Psi$ from $\pi_{1}\left(M_{1}\right)$ to $\pi_{1}\left(M_{2}\right)$ (and thus a bijection between the free homotopy classes of closed curves in $M_{1}$ and $M_{2}$ ) such that the same lengths of closed geodesics occur in corresponding free homotopy classes. The isomorphism $\Psi$ is called $a$ marking.

If $\Phi$ is a $\Gamma$-almost inner automorphism, then by the argument in the proof of Proposition 1.3, we see that $\Phi$ gives a marking between the length spectra of $(\Gamma \backslash G, g)$ and $(\Phi(\Gamma) \backslash G, g)$. The converse holds as well: 
2.4 Proposition. (Eberlein [11]) Given two-step nilmanifolds

$$
M=(\Gamma \backslash G, g) \quad \text { and } \quad M^{*}=\left(\Gamma^{*} \backslash G^{*}, g^{*}\right),
$$

$M$ and $M^{*}$ have the same marked length spectrum if and only if there exists a $\Gamma$-almost inner automorphism $\Phi$ such that $M^{*}$ is isometric to $(\Phi(\Gamma) \backslash$ $G, g)$. Moreover every marking is of the form $\Psi \circ \Phi$ where $\Psi$ is induced by an isometry from $(\Phi(\Gamma) \backslash G, g)$ to $M^{*}$.

R. Gornet ([14]) has constructed an example showing that the "only if" statement fails for higher-step nilmanifolds.

Before stating our results on geodesic flows, we need to take a closer look at almost inner automorphisms (see [12], [13]).

2.5 Definition. A derivation $\varphi$ of a Lie algebra $\mathfrak{g}$ is said to be almost inner if $\varphi(X) \in[X, \mathfrak{g}]$ for all $X \in \mathfrak{g}$, i.e., there exists a map $B: \mathfrak{g} \rightarrow \mathfrak{g}$ (not necessarily continuous) such that $\varphi(X)=[B(X), X]$.

If $\varphi$ is an almost inner derivation of a two-step nilpotent Lie algebra $\mathfrak{g}$, then $\varphi^{2}=0$ and thus $I d+\varphi=\exp (\varphi)$ is an automorphism of $\mathfrak{g}$. Under the isomorphism $\Phi \rightarrow \Phi_{*}$ between automorphisms of a simply-connected Lie group and automorphisms of its Lie algebra, we have :

2.6 Lemma. Let $G$ be a simply-connected two-step nilpotent Lie group. An automorphism $\Phi$ is almost inner if and only if $\Phi_{*}=I d+\varphi$ where $\varphi$ is an almost inner derivation of the Lie algebra $\mathfrak{g}$ of $G$. In the notation of 2.5, we then have

$$
\Phi(\exp X)=a_{X}(\exp X) a_{X}^{-1}, \quad \text { where } a_{X}=\exp (B(X))
$$

The analogous statement holds for $\Gamma$-almost inner automorphisms but then $\varphi$ is only " $\Gamma$-almost inner" in the sense that $\varphi(X) \in[X, \mathfrak{g}]$ for those $X$ such that $\exp (X) \in \Gamma$.

We remark that the mapping $B$ in Definition 2.5 can't be continuous at 0 unless $\varphi$ is inner. Indeed linearity of $\varphi$ implies that $\varphi(X)=[B(t X), X]$ for all non-zero $t \in \mathbf{R}$. If $\varphi$ were continuous at 0 , we would then have $\varphi(X) \equiv[B(0), X]$. A similar argument shows that $B$ can't be continuous at any point in the center of $\mathfrak{g}$ unless $\varphi$ is inner.

2.7 Definition. Let $\mathfrak{g}$ be a two-step nilpotent Lie algebra and let $\mathfrak{v}$ be a vector space complement of $[\mathfrak{g}, \mathfrak{g}]$. Note that $[\mathfrak{g}, \mathfrak{g}]$ is central and thus any almost inner derivation is zero on $[\mathfrak{g}, \mathfrak{g}]$. We will say an almost inner derivation $\varphi$ of $\mathfrak{g}$ is of continuous type if the map $B$ in 2.5 can be chosen to be continuous on $\mathfrak{v}-\{0\}$. We will also say the corresponding almost 
inner automorphism $\Phi$ of the associated simply-connected Lie group $G$ is of continuous type (see Lemma 2.6).

We can now give a more precise statement of Theorem B in the introduction.

2.8 Theorem. Suppose a two-step nilmanifold $M=(\Gamma \backslash G, g)$ and a nilmanifold $M^{*}=\left(\Gamma^{*} \backslash G^{*}, g^{*}\right)$ have $C^{0}$-conjugate geodesic flows. Then $M^{*}$ is isometric to $(\Phi(\Gamma) \backslash G, g)$ for some almost inner automorphism $\Phi$ of continuous type.

Thus the set $\mathcal{M}$ in Theorem B can be taken to be all $(\Gamma \backslash G, g)$ such that $G$ admits no almost inner automorphisms of continuous type other than the inner ones. (Note that if $\Phi$ is inner, then $(\Phi(\Gamma) \backslash G, g)$ is isometric to $(\Gamma \backslash G, g)$.) We will enlarge $\mathcal{M}$ below.

\subsection{Examples.}

i) Many two-step nilpotent Lie groups admit no almost inner automorphisms except for the inner ones. This is true, for example, for the Heisenberg groups, i.e., the two-step nilpotent groups with one-dimensional centers.

ii) By the comments preceding Definition 2.7, no Lie group whose center properly contains its derived group can admit a non-inner almost inner automorphism of continuous type.

iii) (see [13]). Let $G$ be the six-dimensional simply-connected nilpotent Lie group with Lie algebra

$$
\mathfrak{g}=\operatorname{span}\left\{X_{1}, X_{2}, Y_{1}, Y_{2}, Z_{1}, Z_{2}\right\}
$$

with

$$
\left[X_{1}, Y_{1}\right]=\left[X_{2}, Y_{2}\right]=Z_{1}, \quad\left[X_{1}, Y_{2}\right]=Z_{2}
$$

and with all other brackets of basis vectors trivial. The almost inner derivations of $\mathfrak{g}$ are the linear maps which send $X_{2}$ and $Y_{1}$ to multiples of $Z_{1}$, send $X_{1}$ and $Y_{2}$ into $\operatorname{span}\left\{Z_{1}, Z_{2}\right\}$ and send $Z_{1}$ and $Z_{2}$ to 0 . These form a 6 -dimensional space containing the inner derivations as a four-dimensional subspace. The derivations $\varphi_{1}$, respectively $\varphi_{2}$, which send $X_{1}$, respectively $Y_{2}$, to $Z_{2}$ and send all other basis vectors to zero span a two-dimensional family of non-inner almost inner derivations. No almost inner derivation of $\mathfrak{g}$ is of continuous type. If $M=(\Gamma \backslash G, g)$ is any nilmanifold associated with $G$, then there exists a continuous two-parameter family of manifolds strongly Laplace isospectral to $M$ but not $C^{0}$ geodesically conjugate. 
iv) Following Eberlein ([11]), we will say a two-step nilpotent algebra $\mathfrak{g}$ is surjective if for every $X \notin[\mathfrak{g}, \mathfrak{g}]$, the image of $\operatorname{ad}(X)$ is all of $[\mathfrak{g}, \mathfrak{g}]$. In this case it is straightforward to show that every linear map which maps $\mathfrak{g}$ into $[\mathfrak{g}, \mathfrak{g}]$ and sends $[\mathfrak{g}, \mathfrak{g}]$ to zero is almost inner of continuous type. (The authors would like to thank P. Eberlein for showing them this fact.) Unless the center is only one-dimensional, this space of almost inner derivations of continuous type properly contains the almost inner derivations. If $\Gamma$ is any uniform discrete subgroup of the corresponding simply-connected Lie group $G$, then every $\Gamma$-almost inner automorphism of $G$ is almost inner of continuous type.

The converse of Theorem 2.8 does not hold. In fact, the authors know no examples of non-isometric nilmanifolds with $C^{0}$-conjugate geodesic flows.

A. Kaplan ([16]) defined a special class of two-step nilpotent Lie groups with left-invariant metrics $(G, g)$ which he called Lie groups of Heisenberg type. These manifolds have a surprisingly rich and varied geometry and have been studied by several authors (e.g., [11], [24]). We will call any associated (compact) nilmanifold $(\Gamma \backslash G, g)$ a nilmanifold of Heisenberg type. The associated Lie algebras are surjective in the sense of Example 2.9(iv) and thus all Lie groups of Heisenberg type with center of dimension greater than one admit continuous families of almost inner automorphisms of continuous type. However, we have:

2.10 Theorem. If the geodesic flow of a nilmanifold $M$ is $C^{0}$-conjugate to that of a nilmanifold $N$ of Heisenberg type, then $M$ is isometric to $N$.

Thus $\mathcal{M}$ may be enlarged to include the nilmanifolds of Heisenberg type.

We end with a discussion of the geometric ideas underlying Theorem 2.8. The Riemannian metric on $G$ defines an inner product on the Lie algebra $\mathfrak{g}$. Let $S$ be the unit sphere in $\mathfrak{g}$ relative to this inner product. The leftinvariant vector fields give a parallelization both of $T G$ and of $T M$, where $M=\Gamma \backslash G$. In particular the unit tangent bundle $S M$ may be identified with $M \times S$, and $\pi_{1}(S M)$ is isomorphic to $\Gamma$.

Suppose the geodesic flow of $M^{*}$ is $C^{0}$-equivalent to that of $M$, say, $\Psi: S M \rightarrow S M^{*}$ intertwines the flows. The induced isomorphism on fundamental groups, $\Phi: \Gamma \rightarrow \Gamma^{*}$, is then a marking between the length spectra. By Proposition 2.4, after composing $\Phi$ with the differential of an isometry, we may assume that $G=G^{*}, g=g^{*}$ and $\Phi$ extends to a $\Gamma$-almost inner automorphism $\Phi$ of $G$. Thus $M^{*}=(\Phi(\Gamma) \backslash G, g)$.

The Levi-Civita connection for a left-invariant metric satisfies

$$
<\nabla_{X} X, Y>=<[Y, X], X>
$$


when $X, Y \in \mathfrak{g}$. Thus if $X$ is orthogonal to the derived algebra, then $\nabla_{X} X=0$ and the integral curves $h \exp (t X), h \in G$, are geodesics in $G$. (Here exp denotes the Lie group exponential.) Their projections to $M=\Gamma \backslash G$ are then geodesics in $M$.

Let $\mathfrak{v}$ be the orthogonal complement of the derived algebra $[\mathfrak{g}, \mathfrak{g}]$. For simplicity here, we restrict our attention to the special case where $\Gamma$ is compatible with the metric in the sense that $\mathbf{L}:=\{X \in \mathfrak{v} \mid \exp (X) \in \Gamma\}$ is a lattice of full rank in $\mathfrak{v}$. (The ideas in this case go through in general but with considerable complications.)

In our special case ,

$$
S_{\mathbf{L}}:=\left\{X \in S \mid t_{0} X \in \mathbf{L} \quad \text { for some } t_{0} \in \mathbf{R}^{+}\right\}
$$

is dense in the unit sphere $S \cap \mathfrak{v}$. If $X \in S_{\mathbf{L}}$, then the geodesic $\exp (t x)$ descends to a closed geodesic in $M$ in the free homotopy class $[\gamma]_{\Gamma}$, where $\gamma=\exp \left(t_{0} X\right)$ for $t_{0}$ the smallest $t \in \mathbf{R}^{+}$such that $\exp (t X) \in \Gamma$. If $h \in C(\gamma, G)$, then $h \exp (t X)$ also descends to a closed geodesic in this class.

(Side remark: The corresponding closed geodesics in $M$ foliate the submanifold $C(\gamma, \Gamma) \backslash C(\gamma, G)$. This gives a geometric interpretation of the expression $\alpha_{h}(\Gamma)$ in Lemma 1.1.)

The geodesic orbit in the unit tangent bundle $S G=G \times S$ corresponding to the geodesic $h \exp (t X)$ is given by $h \exp (t X) \times\{X\}$. Thus the geodesic orbits in $S G$ which descend to closed orbits in $S M$ in the class $[\gamma]_{\Gamma}$ foliate the submanifold $C(\gamma, G) \times\{X\}$.

(Note: We are being deliberately inaccurate here to avoid minor technical details in the discussion. In particular, the curves $\gamma^{\prime} h \exp (t X), \gamma^{\prime} \in \Gamma$, $h \in C(\gamma, G)$ also project to closed geodesics.)

The geodesic conjugacy $\Psi: S M \rightarrow S M^{*}$ carries closed geodesics in $[\gamma]_{\Gamma}$ to closed geodesics in the free homotopy class $[\Phi(\gamma)]_{\Phi(\Gamma)}$. Since $\Phi$ is a $\Gamma$ almost inner automorphism, there exists $a_{\gamma} \in G$ such that $\Phi(\gamma)=a_{\gamma} \gamma a_{\gamma}^{-1}$. As in the proof of Proposition 1.3, the closed geodesics in $[\Phi(\gamma)]_{\Phi(\Gamma)}$ are the projections of the geodesics $\alpha_{\gamma} \sigma$ in $G$, where $\sigma$ projects to a closed geodesic in the class $[\gamma]_{\Gamma}$ of $M$. The corresponding orbits in $S G$ foliate $a_{\gamma} C(\gamma, G) \times\{X\}$.

We conclude that for each $X \in S_{\mathbf{L}}$ and corresponding $\gamma=\exp \left(t_{0} X\right)$ as above, the lift $\tilde{\Psi}: S G \rightarrow S G$ of the geodesic conjugacy $\Psi: S M \rightarrow S M^{*}$ carries $C(\gamma, G) \times\{X\}$ to $a_{\gamma} C(\gamma, G) \times\{X\}$.

As in 2.5 and 2.6, the automorphism $\Phi$ corresponds to a derivation $\varphi$ of $\mathfrak{g}$ satisfying $\varphi(Y)=[B(Y), Y]$ whenever $\exp (Y) \in \Gamma$, and we have $a_{\gamma}=\exp B\left(t_{0} X\right)$. By linearity, $\varphi(t X)=\left[B\left(t_{0} X\right), t X\right]$ for all $t \in \mathbf{R}$; 
in particular, setting $\tilde{B}(X)=B\left(t_{0} X\right)$, we have $\varphi(X)=[\tilde{B}(X), X]$ for all $X \in S_{\mathbf{L}}$. One now uses the fact that the homeomorphism $\tilde{\Psi}$ maps $C(\gamma, G) \times\{X\}$ to $\exp (\tilde{B}(X)) C(\gamma, G) \times\{X\}$ for all $X \in S_{\mathbf{L}}$ and the density of $S_{\mathbf{L}}$ in $S$ to argue that $\tilde{B}$ extends continuously to $S \cap \mathfrak{v}$ and thus defines a continuous homogeneous map $\tilde{B}: \mathfrak{v}-\{0\} \rightarrow \mathfrak{g}$. By continuity of both $\varphi$ and $\tilde{B}$, we have $\varphi(X)=[\tilde{B}(X), X]$ for all $X \in \mathfrak{v}-\{0\}$. Thus $\Phi$ must be almost inner of continuous type.

\section{Acknowledgements}

The first author would like to thank Hubert Pesce for discussing his work ([22]) and Chal Benson and Gail Ratcliff for an interesting exchange of ideas related to section 1. The authors would like to thank Patrick Eberlein and David Webb for many helpful discussions.

\section{References}

1. P. Bérard, Transplantation et isospectralité, Part I, Math. Ann. 292 (1992), 547559.

2. W. Ballmann, M. Gromov, and V. Schroeder, Manifolds of nonpositive curvature, Birkhauser, Boston, 1985.

3. R. Brooks, P. Perry, and P. Yang, Isospectral sets of conformally equivalent metrics, Duke Math. J. 58 (1989), 131-150.

4. R. Brooks and R. Tse, Isospectral surfaces of small genus, Nagoya Math. J. 107 (1987), 13-24.

5. P. Buser, Isospectral Riemannian surfaces, Ann. Inst. Fourier 36 (1986), 167-192.

6. Y. Colin de Verdière, Spectre du Laplacian et longeur des géodesiques periodiques II, Comp. Math. 27 (1993), 159-184.

7. J. H. Conway and N. J. Sloane, Four-dimensional lattices with the same theta series, Internat. Math. Research Notices (Duke Math. J.) 1992, no. 4, 93-96.

8. C. Croke, Rigidity for surfaces of non-negative curvature, Comm. Math. Helvetici 65 (1990), 150-169.

9. D. DeTurck and C. Gordon, Isospectral deformations II: trace formula, metrics, and potentials, Comm. Pure Appl. Math. 42 (1989), 1067-1095.

10. J. Duistermaat and V. Guillemin, The spectrum of positive elliptic operators and periodic bicharacteristics, Invent. Math. 29 (1975), 30-79.

11. P. Eberlein, Geometry of 2-step nilpotent groups with a left-invariant metric, preprint.

12. C. Gordon, The Laplace spectra versus the length spectra of Riemannian Manifolds, Contemp. Math. 51 (1986), 63-80.

13. C. Gordon and E. N. Wilson, Isospectral deformations of compact solvmanifolds, J. Diff. Geom. 19 (1984), 241-256.

14. R. Gornet, Spectral geometry on higher-step Riemannian nilmanifolds, Ph. D. thesis, Washington University, St. Louis, 1993.

15. _ The length spectrum and representation theory on two and three-step nilpotent Lie groups, to appear in Contemp. Math.

16. A. Kaplan, Riemannian nilmanifolds attached to Clifford modules, Geom. Dedicata 11 (1981), 127-136. 
17. R. Kuwabara, A note on the deformations of Hamiltonian systems on nilmanifolds, J. Math. Tokushima 26 (1992), 19-29.

18. J. Milnor, Eigenvalues of the Laplace operator on certain manifolds, Proc. Nat. Acad. Sci. 51 (1964), 542.

19. J. Otal, Sur les longueurs des géodesiques d'une metrique a courbure negative dans le disque, Comm. Math. Helvetici 65 (1990).

20. H. Ouyang and H. Pesce, Déformations isospectrales sur les nilvariétés de rang deux, C. R. Acad. Sci. Paris, Série I 314 (1992), 621-623.

21. H. Pesce, Calcul du spectre d'une nilvariété de rang deux et applications, to appear in Trans. Amer. Math. Soc.

22. - Variétés hyperboliques et elliptiques fortement isospectrales, preprint.

23. __ Representations relativement équivalentes et variétés riemanniennes isospectrales, preprint.

24. C. Riehm, Explicit spin representations and Lie algebras of Heisenberg type, J. London Math. Soc. 29 (1984), no. 2, 49-62.

25. T. Sunada, Riemannian coverings and isospectral manifolds, Annals of Math. 121 (1985), 169-186.

Department of Mathematics, Dartmouth College, Hanover, Nh 03755 .

E-mail address: carolyn.s.gordon@dartmouth.edu

Department of Mathematics, Texas Tech University, Lubbock, T X 79409 .

E-mail address: ymao@ttmath.ttu.edu 